\title{
OBSERVATIONS ON THE BACTERIOLOGY AND IMMUNE REACTIONS OF RUBEOLA (MEASLES) AND RUBELLA (GERMAN MEASLES)
}

\author{
Plate 14 \\ R UTH T UNNICLIFF \\ From the Memorial Institute for Infectious Diseases, Chicago
}

In a previous article $I^{1}$ have described a micrococcus isolated in anaerobic cultures from the blood of measles patients. The cultures were made during the preeruptive and early eruptive stages of the disease, at which time the virus of measles is present in the blood (Hektoen, ${ }^{2}$ Anderson and Goldberger ${ }^{3}$ ).

The blood was drawn with a sterile syringe from the vein at the elbow, after carefully washing with alcohol or tincture of iodin and alcohol. Generally, whole blood was added to the medium, but sometimes it was collected in sodium citrate solution. The cultures were incubated at $36 \mathrm{C}$.

Anaerobic cultures were made in semicoagulated horse serum; on anaerobic Krumwiede ${ }^{4}$ plates as used by Dick and Henry ${ }^{5}$ in blood cultures in scarlet fever; in ascitic fluid and horse serum alone or with plain and dextrose broth, with and without tissue. Anaerobic cultures were made of laked blood and lately of whole blood in ascitic dextrose agar shake cultures as used by Plot $z^{6}$ in isolating the typhus bacillus.

The coagulated horse serum was prepared by heating the serum, about $10 \mathrm{c} \mathrm{c}$ in each tube, at $56 \mathrm{C}$. for one hour on three successive days, the temperature at last being raised slowly to $70 \mathrm{C}$. and kept there until the serum became semicoagulated. The tubes were incubated four or five days before being used. About $1 \mathrm{cc}$ of blood was added to each tube directly from the syringe and forced toward the bottom of the tube with a sterile pipet. When the cultures were examined, some of the contents from different parts of the tube were removed with a pipet, and smears and subcultures made.

Received for publication Dec. 12, 1917.

1 Jour. Am. Med. Assn., 1917, 68, p. 1028.

2 Jour. Infect. Dis., 1905, 2, p. 238.

- Pub. Health Rep., 1911, 26, 847, 887. Jour. Am. Med. Assn., 1911, 57, p. 113.

- Jour. Infect. Dis., 1913, 12, p. 199.

s Ibid., 1914, 15, p. 85.

- Ibid., 1915, 17, p. 1. Baehr: ibid., 1917, 20, p. 201. 
In using the Plotz method only $0.5 \mathrm{c} c$ of blood was added to the ascitic dextrose agar on account of the difficulty of otherwise seeing the colonies. Large test tubes containing $20 \mathrm{cc}$ of dextrose agar were employed so that the contents could be mixed without being poured into another tube. Aerobic cultures were made in the fluid mediums mentioned. Subcultures from the various inoculations were made on ascitic goat blood-agar slants, in semicoagulated horse serum and various fluid mediums. The original cultures and subcultures were made anaerobic by the use of pyrogallic acid and sodium hydroxid, the stoppers being paraffined. Occasionally sterile fluid paraffin was used on fluid cultures.

Successful cultures were not obtained in any of the anaerobic fluid mediums or in the aerobic cultures. The largest number of positive cultures were obtained in semicoagulated horse serum and the whole blood ascitic dextrose-agar shake cultures. The anaerobic plates and the hemolyzed blood ascitic dextrose shake cultures were fairly satisfactory.

Blood cultures were made from 50 patients in the preeruptive and eruptive stages and from 5 when the rash was fading. In every instance it concerned true measles - rubeola. A small micrococcus was observed in the original anaerobic cultures in 42 of the early cases. The cocci were found in all of the last 15 cases studied. In the earliest culture, obtained a few hours after the appearance of the Koplik spots and 24 hours before the eruption, there were only a few organisms. Cocci were present in large numbers in the cultures of three patients made a few hours before the appearance of the exanthem; two in ascites dextrose shake cultures showed innumerable colonies. Similar shake cultures made in a case when the rash was just appearing showed about one-half as many colonies. The organisms now become less numerous and disappear from the blood as soon as the rash begins to fade.

Twenty-eight of the 42 positive cultures grew slightly for two or three generations only. One strain remained strictly anaerobic, growing only on blood agar and not in fluid mediums. Thirteen strains became aerobic in the second generation and grew fairly abundantly.

Growth appears in the original tubes in from 1-22 days, generally in from 5-15 days. The cultures in which the organisms were first found in the second and third week may have grown out earlier and not been observed. Subcultures generally grow in 24 hours after growth is established. Growth in the strictly anaerobic strain occurs on about the ninth day.

Successful subcultures on plain blood agar plates show small greenish colonies with regular margins giving generally a decidedly green color to the medium. 
The growth on dextrose blood agar is more profuse and more moist and gives the culture a burnt appearance. Some strains produce a slight hemolysis after cultivation. Four of the strains gave larger, moister colonies. These four do not decolorize milk; some of the others produce acidity and some coagulation. They all grow on plain agar as a colorless growth. In ascitic dextrose broth the growth is flocculent, the fluid often remaining clear. They grow a little in plain broth. All ferment dextrose and saccharose and all but one maltose. An occasional strain ferments mannite and lactose. None ferment inulin, salicin and raffinose. None are soluble in bile. None liquefy gelatin.

In smears from the original culture, the organism appears as a very small, round, sometimes flattened diplococcus or in short chains, sometimes as clumps of cocci of varying sizes. In the coagulated horse serum they are so small and sometimes so few in number that considerable search is necessary to find them. They are perfectly distinct, however, when they are found. In subcultures the organisms appear larger, generally as a diplococcus, sometimes in short chains, but sometimes in long chains or in clumps like staphylococci. The cocci at the ends of chains are occasionally smaller than those in the center. This is especially noticed in the anaerobic strains. Large forms are occasionally seen. The organism is gram-positive and stains with ordinary stains. Carbol-gentian-violet was used in staining the original cultures on account of its penetrating power.

A similar diplococcus was isolated on aerobic blood plates from the throats of all the patients examined and was the organism most frequently found in anaerobic cultures from the nose and eye. It was isolated in pure culture from discharge from the ear. The organism was not found in similar blood cultures from five measles patients with fading eruptions, five normal persons, two suspected measles cases and three scarlet fever patients. It is not described by Dick and Henry in their exhaustive work on anaerobic blood cultures in scarlet fever.

Cultures of the diplococcus pass through Berkefeld $\mathrm{N}$ filter. They resist drying 24 hours, none being alive in 48 . Swabs were soaked in a horse serum dextrose broth culture, allowed to dry in sterile test tubes at room temperature for varying periods and then cultured. The organism is killed by heating at $57 \mathrm{C}$. for 45 minutes. It resists a temperature of $-2 \mathrm{C}$. for at least 14 days, and $50 \%$ glycerol 2 months at least.

In scarlet fever, Dick and Henry found that at the height of the attack a variety of bacteria are present in the blood. The same occurs in measles. Before the eruption the diplococci I describe were found 
alone in the blood and alone also in twenty-one cases soon after the appearance of the exanthem. At the height of the fever, however, various other bacteria were isolated from the blood in twenty cases: Aerobic (ten cases) and anaerobic diphtheroid bacilli (twelve), filamentous organisms (six), gram-negative spirilla (four), a black pigment producing bacillus (one), spore forming large bacilli (four), and staphylococci (three). With the exception of the gram-negative spirilla, similar organisms were also cultivated from the throat, nose or eye. It is possible that some of these growths came from contaminations, but surely not all.

The special points of interest in this work were: the almost constant presence of a diplococcus in the blood of measles patients during the pre-eruptive and eruptive stages; its presence alone and in large numbers before the apearance of the eruption and alone also in a large proportion of cases at the height of the infection, and its presence at the same time in the throat, nose and eyes.

This coccus is pathogenic for the rabbit, young dog, mouse, rat, and monkey. The sediment of about $20 \mathrm{cc}$ of horse serum dextrose broth cultures killed a rabbit in 24 hours, the organism being isolated from the heart blood and kidney. No lesions were found. The growth from one blood-agar slant killed a rabbit in 24 hours producing hemorrhages in the heart valve and pneumonia of one lung. Proportional doses killed a mouse in 24 hours and made a young dog and rat ill, but did not kill. A monkey (giant rhesus) was inoculated intravenously with the sediment of $100 \mathrm{cc}$ horse serum dextrose broth. It was very ill the next day and slightly ill for several days, but at no time did it show any symptoms of measles. The leukocyte count rose from 14,000 to 24,000 the day following the inoculation, falling to 7,000 on the 11th day-the same blood picture as produced by the inoculation of measles blood (Hektoen and Eggers, Lucas and Prizer, ${ }^{8}$ Tunnicliff ${ }^{9}$ ). This reaction is considered due to the coccus, because two other monkeys inoculated with equaly large numbers of spirilla isolated from the blood of measles patients produced no such change.

Cocci similar to those found in these blood cultures were found in sections of the lung and kidney in one fatal case of measles; in the lung and bronchial lymph gland of another, and in the gangrenous tongue of a third. They appear singly, in pairs and chains. Some are found inside of leukocytes. The cocci in these sections vary considerably in size (Fig. 3).

Similar blood cultures were made from ten rubella patients. The anaerobic Krumwiede plates, as used by Dick and Henry, gave the

7 Jour. Am. Med. Assn., 1911, 57, p. 1833.

8 Jour. Med. Research, 1912, 26, p. 181.

- Jour. Infect. Dis., 1912, 11, p. 474. 
most satisfactory results. The inverted bottom of a petri dish is placed inside the inverted top and sterilized in a towel, by dry heat. About $1 \mathrm{cc}$ of freshly drawn blood is added to $9 \mathrm{c} \mathrm{c}$ of melted agar, and after thoroughly mixing, this is poured into the inverted top of the petri dish and the inverted bottom laid directly on the melted agar. Boiling paraffin is used to seal the space between the plates. Plain or dextrose agar without ascitic fluid was used. A fairly large diplococcus was observed in the anaerobic cultures in seven of these ten cases. Two cultures made just as the eruption was appearing did not grow in subculture, those from five others, made at the height of the infection, grew fairly abundantly. Two negative cultures were taken from patients at the onset of the attack and one as the eruption was fading. Only one or two colonies were present on each plate in the successful cultures. Similar diplococci were also found in asciticdextrose shake and in semicoagulated horse serum cultures. Single colonies of the same diplococci were found in two cases on the aerobic plates. A diphtheroid bacillus was isolated twice from anaerobic cultures. Throat smears and cultures on aerobic blood-agar plates from six cases showed diplococci similar to those isolated from the blood.

The colonies appear on the anaerobic plates after 1-5 days' incubation, as indistinct greenish-brown colonies. The organism becomes aerobic in the second generation. On plain blood-agar aerobic plates the colonies are small, dull and green with regular margins. They become larger and moister on cultivation. Some strains show slight hemolysis. They produce a burnt appearance on dextrose blood agar. All ferment maltose, dextrose, lactose, salicin, and saccharose. Two strains also ferment inulin, mannite and raffinose. All but one liquefy gelatin, and produce a delicate colorless growth on plain agar. All grow a little in plain broth, one produces a sediment. They grow well in dextrose broth. The organisms are not soluble in bile.

In smears these organisms appear fairly large (1-2 microns in length), in pairs or chains with pointed ends generally, the body often being elongated, occasionally they occur in clumps. The pointed ends disappear on cultivation. They possess a capsule. The diplococcus is gram-positive and stains with the ordinary stains. The cultures of this organism do not pass through a Berkefeld $\mathrm{N}$ filter. They resist drying five days. They are killed by heating at $65 \mathrm{C}$. for one-half hour. They resist a temperature of $-2 \mathrm{C}$. and $50 \%$ glycerin for 6 months at least. They are not pathogenic for rabbits. 
Various immunity tests have been made with the cocci isolated from rubeola and rubella to determine if any antibody reaction could be demonstrated. So far opsonins have been the only antibodies found in any appreciable amount. Both the Wright and the dilution method have been used with similar results. In the dilution method the normal and immune serums are diluted with physiologic sodium chlorid solution to determine the point of opsonic extinction - the point at which induced phagocytosis recognizably exceeds phagocytosis with salt solution. Normal human leukocytes were used. The specimens were incubated at $37 \mathrm{C}$. for 15 minutes and the smears then stained with carbol-thionin. Typical strains of Streptococcus viridans from the throat, pneumococci, and the coccus from poliomyelitis were also studied to determine if there was any specificity in the opsonic action.

The serum of twelve measles patients was examined, in six only once as the eruption was beginning to fade, in six usually daily throughout the course of the disease. Four of these serums showed a negative phase of $1,2,2$, and 5 days' duration, respectively. A distinct rise in opsonins for the measles organism occurred in each case as the symptoms subsided and the eruption disappeared. This rise lasted from 1-4 days. The point of opsonic extinction for normal blood was 1:30; for measles serum 1:15 during the negative phase and from $1: 60$ to $1: 340$ during the rise. The various strains isolated from the blood, throat, nose, eye and ear of measles patients gave similar results.

Two of these patients were examined daily for opsonins with the measles coccus and also with the coccus from the blood of a rubella patient. While the opsonins changed for the measles coccus, they remained within the normal limits for the rubella organism. Five rubella patients showed just the reverse opsonic content in the blood, showing typical opsonic changes for the rubella coccus and none for the measles organism. With the rubella coccus, the point of opsonic extinction for normal serum was $1: 30$; for rubella serum $1: 15$ during the negative phase and $1: 120$ to $1: 240$ during the rise. The negative phase and rise both lasted from 1-3 days. The point of opsonic extinction with the measles coccus with both normal and rubella serum was about $1: 30$. There were no changes in the opsonic content of the blood of either measles or rubella patients for the Streptococcus viridans, pneumococcus or the poliomyelitis coccus, in any of the serums examined. The results indicate that specific opsonins for the rubeola 
and rubella cocci develop during the course of these diseases, disappearing with the recovery of the patient. After cultivation for a variable length of time both the rubeola and rubella cocci lose their original morphological characteristics, but retain their specific immunological properties.

\section{SUM MARY}

Diplococci may be isolated from the blood of patients with rubeola and rubella. While the two organisms are similar in their cultural characteristics, they differ in size, shape, capsule formation, filtrability. viability, virulency, and immunity reactions.

\section{EXPLANATION OF PLATE 14}

Fig. 1.-Forty-eight hour growth on ascitic goat blood agar of diplococcus isolated from blood of rubeola patient; Gram stain; $\times 1000$.

Fig. 2.-Twenty-four hour growth on goat blood agar of diplococcus isolated from throat of rubeola patient; Gram stain; $\times 1000$.

Fig. 3.-Section through lung of rubeola patient showing cocci in chains; Gram stain; $\times 1000$.

Fig. 4.-Twenty-four hour growth on goat blood agar of diplococcus isolated from blood of rubella patient; Gram stain; $\times 1000$.

Fig. 5.-Twenty-four hour growth on goat blood agar of diplococcus isolated from throat of rubella patient; Gram stain; $\times 1000$. 
Plate 14
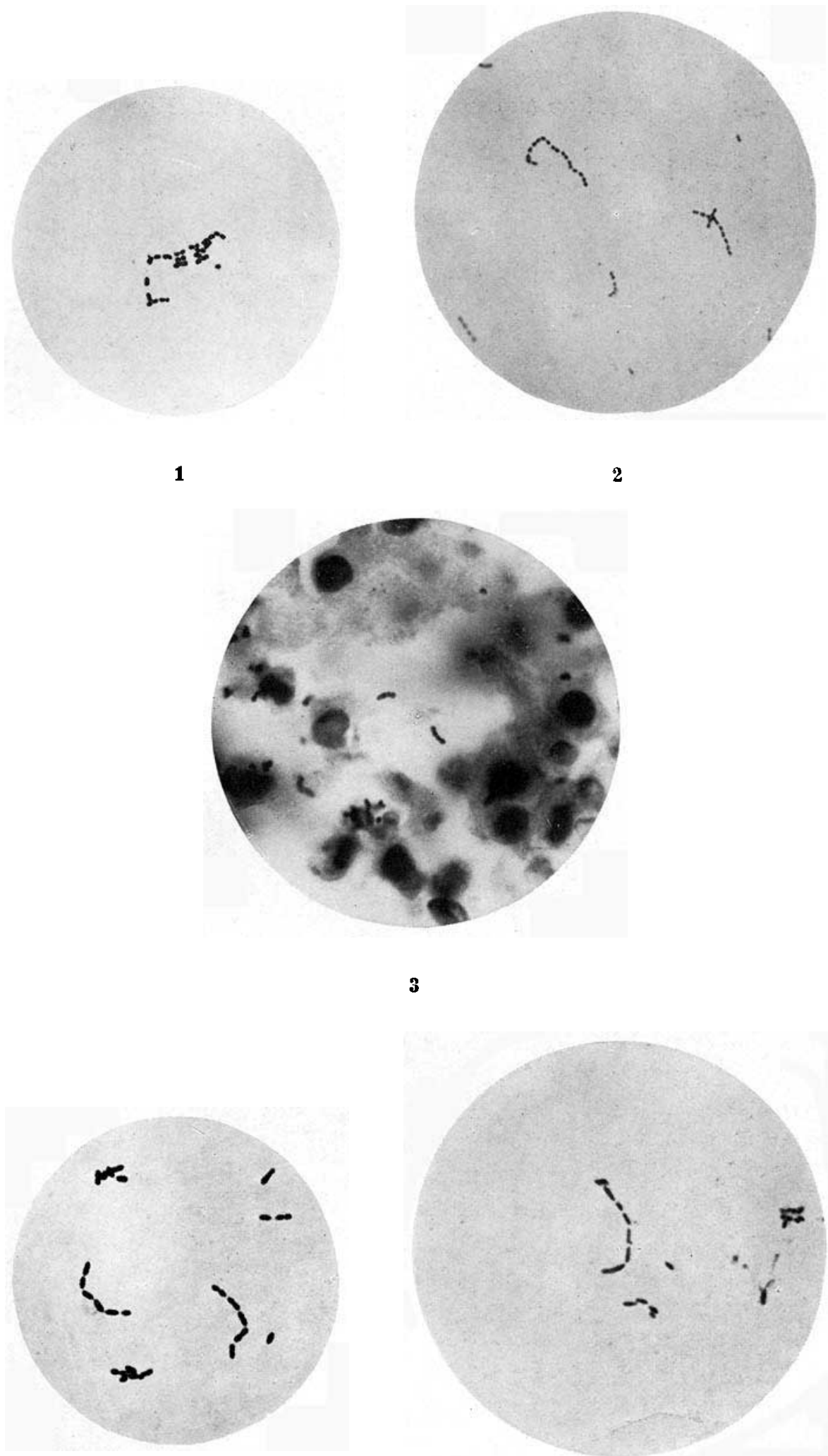

3

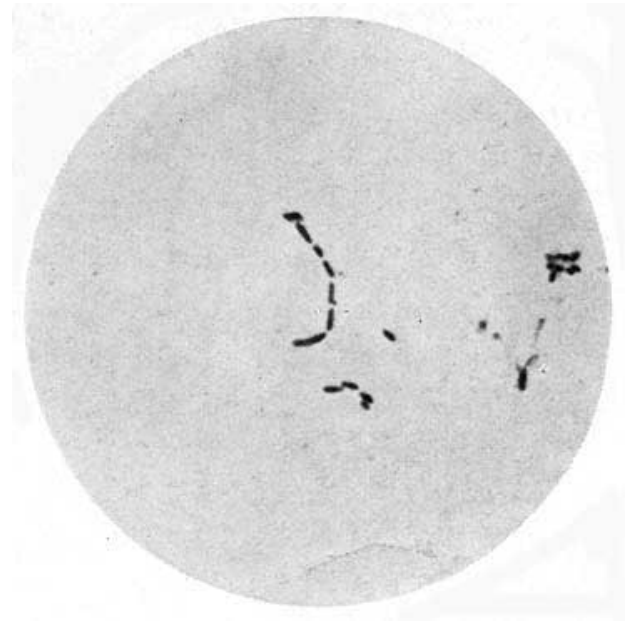

